\title{
Editorial to the Topical Collection: Ocean Worlds
}

\author{
Athena Coustenis ${ }^{1} \cdot$ Rafael Rodrigo $^{2}$ - Tilman Spohn ${ }^{3}$. \\ Jonas L'Haridon ${ }^{4}$
}

Published online: 20 April 2020

(c) Springer Nature B.V. 2020

We are at the beginning of a new era in the exploration of the outer solar system. Spacecraft have already visited each of the giant planets and made detailed observations of their major satellites. In 2017, the Cassini spacecraft ended its highly successful mission in the Saturnian system while the Juno mission has been investigating Jupiter since 2016. The New Horizons spacecraft revealed the Pluto/Charon system in 2015 and is now exploring the Kuiper belt. In parallel, NASA, ESA, and other international space agencies are considering the next major steps in the outer solar system exploration. Among these, the Jupiter Icy moon Explorer (JUICE), the first Large class mission of the ESA Cosmic Vision 2015-2025 campaign currently in development, will explore three satellites of the Jovian system - Ganymede, Europa and Callisto - to study the emergence of habitable worlds around gas giants. NASA is also

Ocean Worlds

Edited by Athena Coustenis, Tilman Spohn, Rafael Rodrigo, Kevin P. Hand, Alexander Hayes, Karen Olsson-Francis, Frank Postberg, Christophe Sotin, Gabriel Tobie, Francois Raulin and Nicolas Walter

\footnotetext{
A. Coustenis

Athena.coustenis@obspm.fr

R. Rodrigo

r.rodrigo@cab.inta-csic.es

T. Spohn

tilman.spohn@issibern.ch

J. L'Haridon

jlharidon@esf.org

1 Laboratoire d'Etudes Spatiales et d'Instrumentation en Astrophysique (LESIA), Paris Observatory, CNRS, Université PSL, Sorbonne Université, Université de Paris, 5, place Jules Janssen, 92195 Meudon Cedex, France

2 Centro de Astrobiología (INTA-CSIC), European Space Astronomy Center (ESAC), Camino Bajo del Castillo s/n, Urb. Villafranca del Castillo, 28692 Villanueva de la Cañada, Madrid, Spain

3 International Space Science Institute, Hallerstrasse 6, 3012 Bern, Switzerland

4 European Science Foundation, 1 quai Lezay-Marnésia, 67080 Strasbourg Cedex, France
} 
developing the Europa Clipper mission to explore the eponymous moon of Jupiter through several flybys and investigate the existence and characteristics of a water ocean covered by an ice shell. NASA also recently selected the Dragonfly quadricopter concept as the next New Frontiers mission (NF4) to investigate Titan. In addition, several other proposals with similar objectives and exciting concepts have been or will be submitted to space agencies in response to future calls.

These investigations all tie into the astrobiological significance of the outer solar system. In looking for (the origin of) life in habitable worlds, the criteria to consider are liquid water, CHNOPS elements (carbon, hydrogen, nitrogen, oxygen, phosphorus and sulfur), energy sources and a stable environment. These requirements are notably met on Earth close to hydrothermal vents on the seafloor, which likely constituted the cradle of life about 4 billion years ago. Ignored for some time to the benefit of the inner solar system objects (Mars essentially), the more remote provinces of our solar system are now being scrutinized with renewed interest for astrobiological purposes and the reasons behind this shift in paradigm are manifold. First, organic materials are present in considerable abundances across the outer solar system. While this is particularly true for Titan and Enceladus around Saturn, the spectral characteristics of the tenuous atmospheres around many other icy and rocky bodies suggest the presence of organic compounds. Second, many satellites of the giant planets are still geologically active today. This is apparent on several large moons such as Io (silicate volcanism), Europa (youthful and complexly modified surface), Ganymede (active magnetic field implying a hot core), Titan (active hydrological cycle based on methane and aeolian processes), and Triton (active geysers). Moreover, geological activity occurs even on tiny Enceladus (active venting from the south polar region). Third, it has been demonstrated that a large number of icy satellites could possess (or have possessed in the past) subsurface liquid water. Consequently, it is possible that the surfaces and interiors of many outer solar system bodies have undergone significant chemical processing over the last 4.5 billion years. While the most robust evidence for liquid water pertains to Europa, subsurface liquid water could also be present on Ganymede, Callisto, Titan, Triton, and Enceladus, if not on most outer solar system icy bodies. In that regard, major clues to understanding the evolution of icy satellites are likely to be found on other satellites as well as on objects of the Kuiper belt. Lastly, another important factor to be considered for the emergence and development of life is that organic compounds have to be present in large enough concentrations (as in the pores of hydrothermal vents) to allow for the formation of more complex molecules, which constitute the necessary building blocks of life. This is not trivial since, on these satellites, life will not emerge in so-called "primordial soups" resulting from Miller-type experiments or the "raining out" of atmospheric organic compounds. Indeed, these "ocean soups" are too large to favor interactions between molecules in such a diluted medium.

The combined presence of water, organic materials and energy sources on some of these outer solar system bodies suggest that exploring them further will likely yield significant results pertinent to astrobiology and to our understanding of the habitable conditions in the solar system, including potential clues on the emergence of life and its survival on our planet. Moreover, recent discoveries could challenge our perception of necessary planetary protection policies for the outer solar system bodies https://cosparhq.cnes.fr/scientific-structure/ panels/panel-on-planetary-protection-ppp/.

Determining the astrobiological potential of other planetary systems, or the range of habitable conditions within the solar system requires an investigation of the origin and evolution of these outer solar system objects, the chemical processing they experienced, and their potentially habitable environments. In addition, the architecture of the outer solar system itself has important implications for astrobiology: the nature and composition of the giant planets, 
their locations in the solar system, their relationship to the Kuiper-belt objects, and the properties of planetary rings all help us to understand the processes involved in the evolution of the solar system.

Implications from astrobiological studies of the outer solar system also extend beyond the confines of our planetary system. Current evidence suggests that innumerable icy worlds around giant planets exist beyond the solar system, any one of which could harbor habitable environments. In turn, many worlds that were once like Titan or Europa must surround parent stars that have now become red giants, raising temperatures to the point where formerly icy worlds could now support liquid water at their surfaces.

In order to make progress in our investigations of these subjects, future missions to the so-called "ocean worlds" are mandatory and both ESA and NASA have incorporated the possibility for such missions in their space programs. For instance, the 2016 budget proposal from the U.S. House Appropriations Committee calls for the creation of an "Ocean Worlds Exploration Program" which would support new missions to Europa, Enceladus, and Titan. This new program enables the investigation of these worlds within the "Vision and Voyages for Planetary Science in the Decade 2013-2022” Decadal Survey and has, therefore, received positive feedback from the scientific community.

Admittedly, the study of our own planet's oceans and their interactions with the atmosphere also offer important insights into the investigation of foreign ocean worlds. Thus, to consolidate the interrogations and findings from the exploration of both terrestrial and extraterrestrial oceans, the ESSC Committee from the European Science Foundation (ESF-ESSC: essc.esf.org) initiated an interdisciplinary study on "Ocean Worlds" in collaboration with the European Marine Board (https://www.marineboard.eu/), the International Space Science Institute (ISSI: https://www.issibern.ch/) and National Academy of Sciences / Space Studies Board (https://www.nationalacademies.org/ssb/space-studies-board) experts.

This collaborative effort started with an ISSI Workshop organized in Bern, Switzerland, from 18 to 22 June 2018. There, 44 international experts gathered to review and synthesize what is presently known on the astrobiological potential of the outer solar system (over 39 presentations), resulting in an intensive and stimulating experience. The contributions and discussions from the workshop led to the publication of a collection of thematic scientific papers regrouped in the "Ocean Worlds" Topical Collection in Space Science Reviews and co-published as a volume of the Space Sciences Series of ISSI (SSSI) by Springer Verlag.

As such, the purpose here is to present a state-of-the-art collection of scientific papers of our understanding of "ocean worlds" - i.e. places that have the potential to harbor subsurface liquid water oceans - and their astrobiological potential, since life likely emerged in analog environments on Earth 4 billion years ago. The review papers in this issue thus provide an overview of the key scientific questions, interpretations and measurements for the exploration of ocean worlds known today, in parallel to a comprehensive review of under-study and novel mission concepts. They cover topics which could be of interest to space agencies in order to establish a strategy for the future exploration of the outer solar system and its astrobiological potential.

Hand et al. review the conditions that need to be met for ocean worlds to be classified as habitable and provide a framework for their systemic exploration in the future.

Campubri-Casas et al. provide a critical overview of the possible scenarios for the emergence of life on Earth, from geological, biological and chemical perspectives, and discuss whether similar processes could have occurred on any of the oceanic icy moons of the solar system.

Journaux et al. delve into the specific thermodynamics, dynamic regimes and evolution scenarios of large ocean worlds - on which high-pressure ice polymorphs form - and discuss 
the implications for habitability, extrapolations to exoplanetary ocean worlds and perspectives for future space exploration missions.

Soderlund et al. review the potential exchange processes between the surface and the interiors of icy moons, which are based on numerical models of ice shell and ocean dynamics and terrestrial analog studies, and their implications for habitability

De Sanctis et al. argue for the existence of relict ocean worlds, taking Ceres as a particularly instructive example, and discuss the implications that many large icy worlds could harbor or could have harbored in the past deep oceans.

Taubner et al. aim to bring together studies and experimental designs from various scientific fields to provide an overview of promising in situ, in silico, and in vitro experiments for the exploration of the different regions of interest on icy moons (e.g. outgassing plumes, icy shells, water and brines oceans, hydrothermal vents and rocky cores).

Jebbar et al. describe micro-organisms that adapted to survive in extreme environments on Earth, also known as extremophiles, and discuss how their study could provide invaluable insights into the potential astrobiology of ocean worlds and help in the identification of potential biosignatures.

Dachwald et al. discuss the key technologies and instrumentation required for the challenging subsurface exploration of ocean worlds, with a focus on the Europa and Enceladus moons as the most promising candidate destinations for future missions, but also consider simpler alternative mission concepts involving impactors, penetrators and plumefly-throughs.

This collection of papers underlines the importance of the outer solar system and of ocean worlds in general for understanding the habitability of the solar system and beyond. It shows that studies of the terrestrial oceans, theoretical analyses, as well as laboratory measurements and spacecraft missions all tie together in the exploration of ocean worlds and increase our knowledge of our planet, the solar system and beyond.

Publisher's Note Springer Nature remains neutral with regard to jurisdictional claims in published maps and institutional affiliations. 